\title{
Functional Columnar Liquid Crystalline Phases From Ionic Complexes of Dendronized Polymers and Sulfate Alkyl Tails
}

\author{
Nadia Canilho, ${ }^{1}$ Edis Kasëmi, ${ }^{2}$ A. Dieter Schlüter, ${ }^{2}$ Janne Ruokolainen, ${ }^{3}$ \\ Raffaele Mezzenga ${ }^{* 1,4}$
}

\begin{abstract}
Summary: We describe how cationic dendronized polymers of generations 1 , and 2 and anionic monoalkyl tails can be combined by supramolecular ionic complexation into comb-like liquid crystalline polymers. The final structures in bulk of these supramolecular complexes were studied by differential scanning calorimetry (DSC), cross-polarized optical microscopy (CPOM), small angle x-rays scattering (SAXS) and transmission electron microscopy (TEM). The combination of these techniques allowed elucidating (i) that these complexes exhibit thermotropic behaviour, (ii) that various liquid crystalline structures in the $3-5 \mathrm{~nm}$ length scale can be obtained such as columnar rectangular, columnar tetragonal, columnar hexagonal and lamellar, depending both on alkyl tail length and polymer generation, (iii) that although the alkyl tails represent the majority phase in the columnar phases, they form the cylindric domains, and the dendronized polymers occupy the continuous domains. Therefore, upon selective cleavage of the alkyl tails in the columnar phases, the present self-assembly approach may constitute an efficient strategy towards the formation of porous organic matrices with ultra-dense pore size in the range of 2 to $4 \mathrm{~nm}$.
\end{abstract}

Keywords: dendronized polymers; lipids; liquid crystals; self-assembly; supramolecular complexation

\section{Introduction}

Supramolecular ionic complexation between polyeletrolytes and low molecular weight countercharged compounds such as surfactants and mesogenes, has been extensively investigated in the last decade to generate macromolecular complexes with characteristics combining the structural properties of the polyelectrolyte template with the length

\footnotetext{
${ }^{1}$ Department of Physics and Fribourg Center for Nanomaterials, Ch. du Musée 3, CH-1700 Fribourg (Switzerland)

E-mail: raffaele.mezzenga@unifr.ch

${ }^{2}$ Laboratory for Polymer Chemistry, Swiss Federal Institute of Technology, Department of Materials, ETHZurich, HCI J 541, CH-8093, Zurich, (Switzerland)

${ }^{3}$ Helsinki University of Technology, Physics Laboratory, Helsinki, 02015 Finland

${ }^{4}$ Nestlé Research Center, Vers-Chez-les-Blancs, 1000, Lausanne 26 (Switzerland)
}

scales imparted by ionically bound, low molecular weight compounds. ${ }^{[1,2]}$

The final structures and properties of these complexes may be tuned by changing the type of low molecular weight ionic compounds, as well as the complexation ratio of compounds units versus the total number of charges present in the polyeletrolyte. An even more efficient way to design the final structures of the complexes consists in tailoring the molecular architecture of the polyelectrolyte template. The initial works have focused on linear polyelectrolytes and alkyl tail surfactants, ${ }^{[3]}$ and has successively extended to macromolecular polyelectrolytes with diverse and complex molecular architectures such as branched polyeletrolytes, ${ }^{[4]}$ peptidic polymers with secondary $\alpha$-helices structures, ${ }^{[5,6]}$ DNA $^{[7,8,9]}$ or other types of rod-like polyelectrolytes. ${ }^{[10]}$ 
The great majority of these classes of complexes exhibited lamellar phases with the polyeletrolyte sandwiched in between layers of alkyl tails, whose packing configuration depended intimately on the type of polyelectrolyte used, but which was typically found to be either a bilayer or a monolayer of interdigitated chains.

More recently, other types of macromolecular polyelectrolytes have been explored such as dendrimers ${ }^{[11,12]}$ and dendronized polymers, ${ }^{[13,14]}$ leading to richer polymorphism compared to previous systems. In particular it has been shown that for these classes of highly branched systems can form columnar phases of various lattices such as rectangular hexagonal and lamellar types. Nevertheless, since these systems have been studied by Small Angle X-Ray Scattering (SAXS), no real insight has been made possible on the packing mechanisms of the polymer and the alkyl tails.

In the present study we combine real space techniques such as transmission electron microscopy (TEM), with diffraction methods to attribute unambiguously the nature of discrete and continuous domains in the columnar phases and to elucidate the possible packing mechanisms of both the lipid and the polymer components.

\section{Experimental Part}

\section{Materials and Complex Preparation}

Sodium octyl sulfate (C8), sodium dodecyl sulfate (C12), and sodium octadecyl sulfate (C18) (Fluka, 98\%) sulfated surfactant salts were selected for ionic complexation with cationic dendronized polymers and used as received (Fluka, $\mathrm{CH}$ ).

First (PG1) and second (PG2) generation cationic dendronized polymers synthesized as described previously ${ }^{[13,14]}$ were selected to form comb-like ionic supramolecular complexes as depicted in Figure 1. Complexation was realized in water solutions maintained at $\mathrm{pH}=3-4$. Dendronized polymers were dissolved into a water solution, then a second water solution at the same $\mathrm{pH}$, containing a stoichiometric

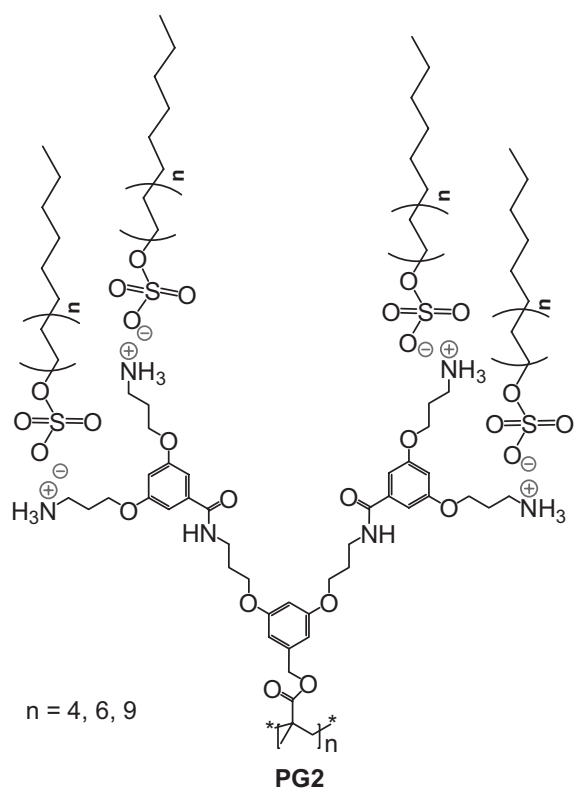

Figure 1.

Schematic representation of a PG2 dendronized polymers complexed with sulfate alkyl tails of varying lengths.

amount of surfactant, was added drop-wise to the first solution, under continuous stirring. The complex removed after precipitation by means of centrifugation, was dried under vacuum at room temperature for three days before analytical and physical characterization.

\section{Annealing Procedure}

The complexes were annealed for three days in a high vacuum column $\left(10^{-8} \mathrm{mBar}\right)$ at temperatures typically between $80^{\circ} \mathrm{C}$ and $150^{\circ} \mathrm{C}$, that is, between the glass transition temperature of the dendronized polymers $(\mathrm{Tg})$ and the temperature of order/disorder transition $\left(T_{O D T}\right)$ of the liquid crystalline structures investigated. This procedure yielded thermodynamic stable structure, as demonstrated by the complete reversibility of phase transitions upon heating/ cooling above/below the $T_{O D T}$.

\section{Small Angle X-rays Scattering}

Small and wide angle $\mathrm{x}$-rays scattering (SWAXS) experiments were performed on a SAXSess instrument with a $\mathrm{CuK} \alpha$ 
X-ray source $(\lambda=1.542$ ̊́) and a slit camera configuration. Samples were placed in between two mica foils within the sample holder and diffraction data were acquired during $30 \mathrm{~min}$. Each diffractogram was acquired after one hour time equilibration at isothermal temperature conditions. The signal arising from mica background was removed from sample measurements to provide the final diffractograms.

\section{Transmission Electron Microscopy}

Ultra thin sections of $40-70 \mathrm{~nm}$ in thickness were cryomicrotomed at $T=-60^{\circ} \mathrm{C}$ using a Leica Ultracut UCT-ultramicrotome and a Diatome diamond knife. Sections were collected onto lacey carbon film coated copper grids. Selective staining of dendronized polymer domains was achieved exposing sections for $20 \mathrm{~min}$ in vapors of aqueous $\mathrm{RuO} 4$ solution. Bright-field TEM was performed on FEI Tecnai 12 transmission electron microscope operating at $120 \mathrm{kV}$. Images were recorded using Gatan Ultra-Scan 1000 camera.

\section{Results and Discussion}

Figure 1 shows, as an illustrative case, the structure of the PG2-C12 complex yielded by the supramolecular ionic complexation of dendronized polymers and sulfate alkyl tail surfactants. It will be shown in what follows that the exact combination of a specific generation with a defined length of the alkyl tail determines precisely the nature and the liquid crystalline structure of the final ionic complex.

The degree of complexation measured by elemental analysis (performed on a Leco
CHNS-932 instrument), proved to be very efficient, since virtually a 1:1 complexation was measured for all the samples investigated, as shown in Table 1, where expected quantities of various elements are compared with experimentally measured quantities.

These findings, which are in agreement with previously reported FTIR and NMR analysis, ${ }^{[13,14]}$ allow a straightforward evaluation of the volume fraction of alkyl tails and dendronized polymers in final complexes, as soon as the densities of the individual components are measured.

The volume fraction of alkyl tails in the final complexes can be formally expressed as:

$$
\phi_{i j}=\frac{\frac{2^{j} M_{i}}{\rho_{i}}}{\frac{M j}{\rho_{j}}+\frac{2^{j} M_{i}}{\rho_{i}}}
$$

given the fact that $2^{j}$ surfactant molecules of mass $M_{i}$ and density $\rho_{i}$ are attached to each repeat unit of the dendronized polymer having mass $M_{j}$ and density $\rho_{j}$.

The densities needed to solve equation 1 were measured by pycnometry on an Accupyc 1330 instrument from Micromeritics. Values of $1.45 \mathrm{~g} / \mathrm{cm}^{3}$ and $1.48 \mathrm{~g} / \mathrm{cm}^{3}$ were measured for PG1 and PG2, respectively, while $1.05,1.09$ and $1.12 \mathrm{~g} / \mathrm{cm}^{3}$ were found for $\mathrm{C} 8, \mathrm{C} 12$ and $\mathrm{C} 18$, respectively. The volume fractions of surfactant in the complexes, as obtained by Equation 1 are reported in Table 2.

The structures in bulk of the dendronized polymer-surfactant complexes were first investigated by cross-polarized optical microscopy and small angle X-ray scattering. Figure 2a shows the diffractogram of

Table 1.

Elemental analysis data of various dendronized polymer-surfactant complexes.

\begin{tabular}{llcccc}
\hline Entry & Complex & C Expected/Found & H Expected/Found & N Expected/Found & S Expected/Found \\
\hline $\mathbf{1}$ & PG1-C8 & $53.35 / 53.42$ & $8.41 / 8.31$ & $3.77 / 3.78$ & $8.63 / 8.55$ \\
$\mathbf{2}$ & PG2-C8 & $54.13 / 53.99$ & $8.12 / 8.09$ & $5.05 / 5.00$ & $7.71 / 7.58$ \\
$\mathbf{4}$ & PG1-C12 & $57.58 / 57.33$ & $9.19 / 9.13$ & $3.28 / 3.29$ & $7.50 / 7.27$ \\
$\mathbf{5}$ & PG2-C12 & $57.87 / 56.80$ & $8.86 / 8.49$ & $4.45 / 4.34$ & $6.79 / 7.07$ \\
$\mathbf{7}$ & PG1-C18 & $62.20 / 61.43$ & $10.04 / 9.87$ & $2.74 / 2.53$ & $6.27 / 5.98$ \\
$\mathbf{8}$ & PG2-C18 & $62.07 / 63.25$ & $9.69 / 9.79$ & $3.78 / 3.32$ & $5.76 / 5.33$ \\
\hline
\end{tabular}


Table 2.

Surfactant volume fractions calculated for each complex, by Equation 1.

\begin{tabular}{llll}
\hline & C8 & C12 & C18 \\
\hline PG1 & 0.57 & 0.62 & 0.67 \\
PG2 & 0.50 & 0.55 & 0.61 \\
\hline
\end{tabular}

dendronized polymer of generation 1 complexed with $\mathrm{C} 8, \mathrm{C} 12$ and $\mathrm{C} 18$, respectively. When $\mathrm{C} 8$ is used, the PG1-C8 complex, which does not exhibit any birefringency, shows a single broad peak in SAXS diffractogram which is typical of systems with short range liquid like order. When the length of the surfactant is increased to $\mathrm{C} 12$, however, three reflections spaced as $\mathrm{q}_{1}: \mathrm{q}_{2}: \mathrm{q}_{3}=1: \sqrt{ } 3: 2$ are observed, which are typical of columnar hexagonal phase (lattice parameter $\mathrm{a}=3.85 \mathrm{~nm}$ ). Finally, when $\mathrm{C} 18$ is complexed with PG1, four reflections spaced as 1:2:3.4 indicate that the system structures into a long-range ordered lamellar phase (lattice parameter $\mathrm{a}=4.22 \mathrm{~nm}$ ). When a PG2 dendronized polymer is complexed with the same sulfate alkyl tails, however,

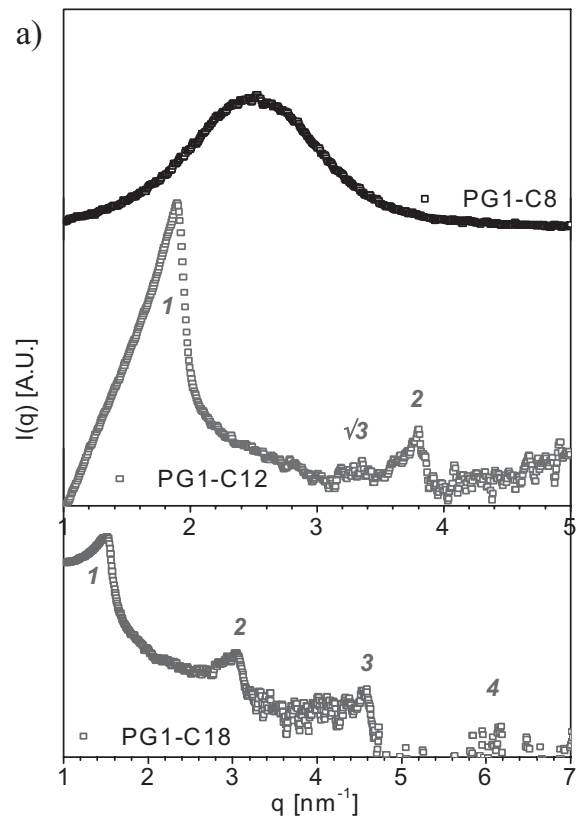

different structures are observed, as shown in Figure 2b. Indeed, when C8 is used for complexation, the PG2-C8 complex becomes birefringent and the corresponding diffractogram exhibits two peaks with ratio $\mathrm{q}_{1}: \mathrm{q}_{2} \approx 1.6$ which can be attributed to a columnar rectangular phase whose lattice has $3.69 \mathrm{~nm}$ and $2.41 \mathrm{~nm}$ square edges. By increasing the alkyl tail length to $\mathrm{C} 12$ two strong reflections and two weak reflections are observed spaced as $\mathrm{q}_{1}: \mathrm{q}_{2}: \mathrm{q}_{3}: \mathrm{q}_{4}$ $=1: \sqrt{ } 2: 2: 3$, which is consistent with a tetragonal phase with lattice period $3.92 \mathrm{~nm}$. Finally, by further increasing the alkyl chain length to $\mathrm{C} 18$, two reflections spaced as $\mathrm{q}_{1}: \mathrm{q}_{2}=1: 2$, indicate a lamellar phase with lattice period $4.62 \mathrm{~nm}$.

The liquid crystalline phases discussed above, exhibit all thermotropic behavior. Figure 3 shows the evolution in the structure factor of the columnar hexagonal phase obtained for PG1-C12 complex at various temperatures. At room temperature, the three reflections typical of the hexagonal phase are all visible, and the sample is birefringent, as expected by

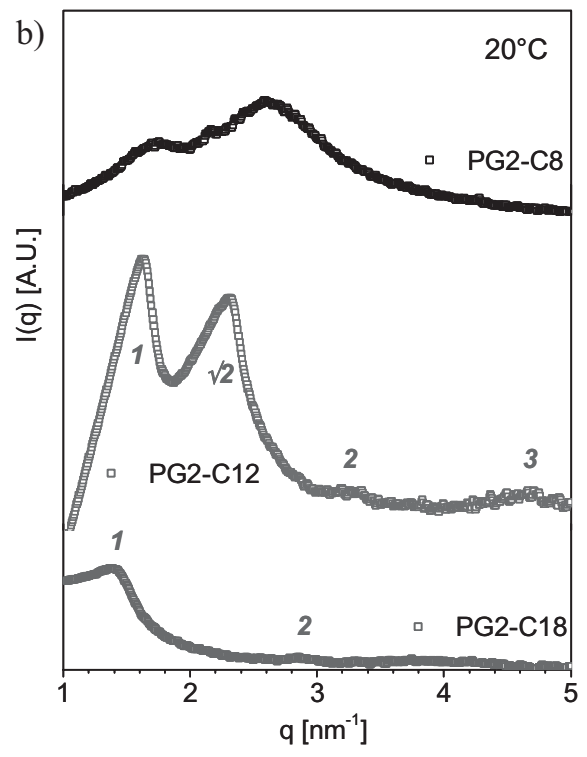

Figure 2.

(a) SAXS diffractograms of PG1 complexed with $\mathrm{C} 8, \mathrm{C} 12$ and $\mathrm{C} 18$. (b) SAXS diffractograms of PG2 complexed with C8, $\mathrm{C} 12$ and $\mathrm{C} 18$. From ref. ${ }^{[14]}$. 


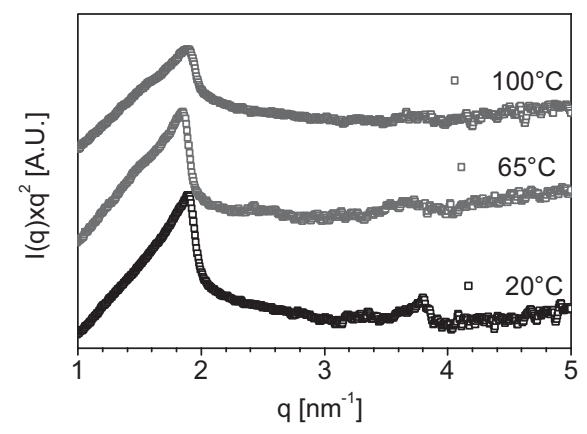

Figure 3.

Diffractograms of the columnar hexagonal phase obtained for $\mathrm{PG} 1-\mathrm{C}_{12}$ complex at various temperatures.

the anisotropy of interfaces in the hexagonal phase. When the temperature is raised to $65^{\circ} \mathrm{C}$, however, the second and third reflections have disappeared and only the first reflection is still visible. Finally, at $100{ }^{\circ} \mathrm{C}$, the first peak has broadened and the sample has completely lost its birefringency, which indicates a short range liquidlike order. The order-disorder transition temperatures of the various complexes investigated here are summarized in Table 3.

The structural analysis based solely on SAXS and cross polarized optical microscopy does not allow establishing univocally the nature of the dispersed and continuous domains of the columnar phases. According to the volume fractions reported in Table 2, one would expect that the alkyl tails form the continuous domains, whereas the minority component, e.g. the dendronized polymer, is expected to occupy the cylinders in all the columnar phases. In order to assess this point unambiguously we have performed transmission electron microscopy on the various complexes investigated. Figure 4 reports

Table 3.

Order disorder transition temperatures for the various liquid crystalline phases observed.

\begin{tabular}{lcc}
\hline $\mathrm{C} 18$ & $65^{\circ} \mathrm{C}$ & $65^{\circ} \mathrm{C}$ \\
$\mathrm{C} 12$ & $85^{\circ} \mathrm{C}$ & $150^{\circ} \mathrm{C}$ \\
$\mathrm{C} 8$ & - & $150^{\circ} \mathrm{C}$ \\
& $\mathrm{PG} 1$ & $\mathrm{PG} 2$ \\
\hline
\end{tabular}

the hexagonal, tetragonal and lamellar structures observed respectively for the PG1-C12, PG2-C12 and PG1-C18 complexes. The staining agent used, RuO4, selectively stains the aromatic groups present in the dendronized polymers, without staining the fully saturated sulfate alkyl tails. ${ }^{[15]}$ As a consequence, in Figure 4 the dendronized polymer appears dark while the surfactant domains appear clear. It can therefore be concluded that, in the columnar phases, the surfactant tails are confined to the cylindrical domains despite the fact that they occupy the majority of the volume fraction, while the dendronized polymers occupy the continuous domains in between the cylinders.

The findings open new scenarios and possible applications for dendronized polymer-alkyl tail complexes in the field on nanopourous materials.

Indeed, because the surfactant occupies the discrete domains, one can envisage selective cleavage of the alkyl tail components without disrupting the lattice structure of the dendronized polymers. Similar concepts have been used by Ikkala et $a l$. to remove by selective solvent, surfactant hydrogen-bonded to one cylindrical domains of a hexagonally packed block copolymer system. ${ }^{[16]}$

In the present case, however, the porous material would be structured over typical lengths scales which are smaller by one order of magnitude compared to previously reported materials, ${ }^{[16]}$ as the lattice parameter is in the range of $2-4 \mathrm{~nm}$. Consequently, the size of pores would also be smaller by one order of magnitude and the density of pores higher by two orders of magnitudes.

Alkyl tails cannot be removed by selective solvents as the ionic binding between ammonium and sulfate charges is markedly stronger than hydrogen bonding used by other authors. ${ }^{[16]}$ Rather, the unbinding between charges can be pursued by de-protonating the ammonium charges when exposing the complexes to basic aqueous environment. This is expected to result on neutralization of ammonium 

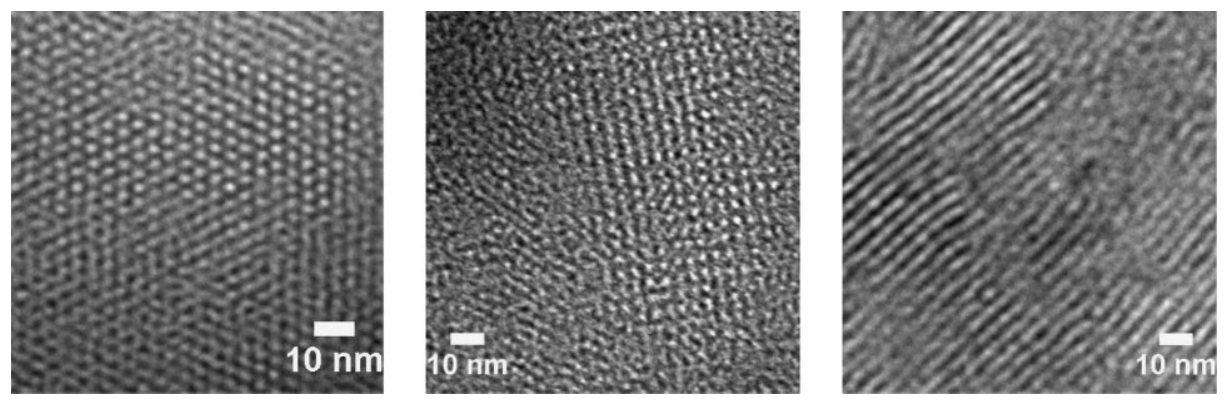

Figure 4 .

Hexagonal, tetragonal and lamellar structures observed respectively for the $\mathrm{PG} 1-\mathrm{C}_{12}, \mathrm{PG}_{2}-\mathrm{C}_{12}$ and $\mathrm{PG} 1-\mathrm{C}_{18}$ complexes. $^{[14]}$

charges and subsequent unbinding between alkyl tails and surfactants. Because at basic $\mathrm{pH}$ uncharged dendronized polymers are non-soluble in water, while sulfate alkyl tail are, the proposed scheme should allow the efficient design of nanoporous materials with ultra high density of pores with nmsize. Figure 5 summaries the main points proposed to achieve nanoporous materials based on dendronized polymer-alkyl tail surfactant complexes.

\section{Conclusions}

We have discussed the self-assembly mechanisms and structures of dendronized polymer-sulfate alkyl tail surfactant complexes as a function of dendronized polymer generation (first and second) and alkyl tail length (from C12 to C18). Depending on the specific combination of generation and alkyl tail used in the complex, small angle $\mathrm{x}$-ray scattering and cross-polarized optical microscopy revealed, on dried and annealed samples, liquid crystalline phases with lamellar structure, as well as a multitude of columnar phases with rectangular, tetragonal and hexagonal lattice. The use of transmission electron microscopy on selectively stained samples allowed the unambiguous attribution of the cylindrical domains to the alky tails. The possible implications of these findings on the design

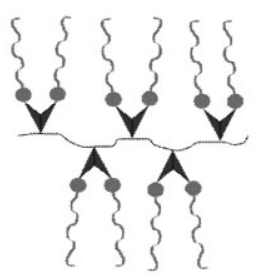

Annealed
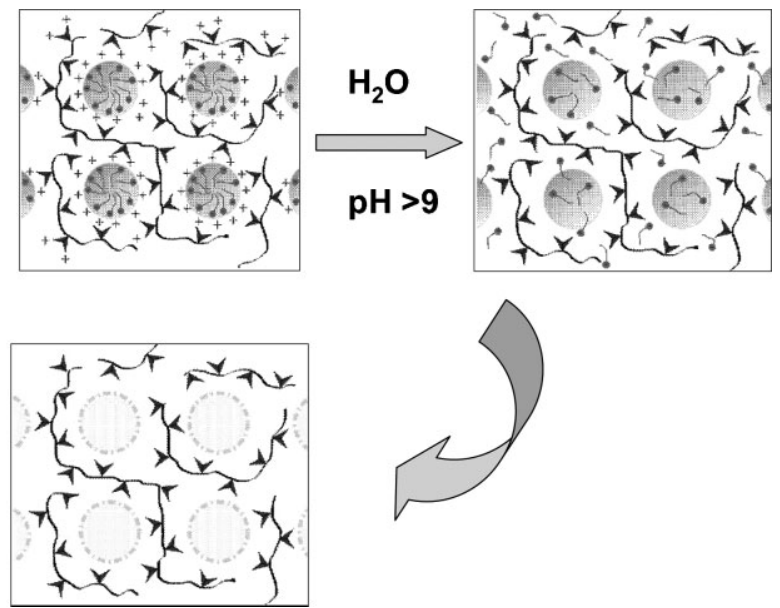

\section{Figure 5.}

Visionary cartoon on the critical steps towards a possible use of dendronized polymer-alkyl tail surfactant complexes as templates for nanoporous materials. 
of potential nanoporous materials with small pore size $(<4 \mathrm{~nm})$ and high density of pores, based on dendronized polymersurfactant templates were discussed in the last section of the paper.

[1] C. F. J. Faul, M. Antonietti, Adv. Mater. 2003, 15, 673. [2] A. F. Thunemann, Prog. Polym. Sci. 2002, 27, 1473.

[3] M. Antonietti, J. Conrad, A. Thunemann, Macromolecules 1994, 27, 6007

[4] B. Y. Ren, Z. Y. Cheng, Z. Tong, X. X. Liu, C. Y. Wang, F. Zeng, Macromolecules 2006, 39, 6552.

[5] E. A. Ponomarenko, A. J. Waddon, K. N. Bakeev, D. A. Tirrell, W. J. MacKnight, Macromolecules 1996, 29, 4340.

[6] W. J. MacKnight, E. A. Ponomarenko, D. A. Tirrell, Acc. Chem Res. 1998, 31, 781.

[7] J. O. Radler, I. Koltover, T. Salditt, C. R. Safinya, Science 1997, 275, 5301.
[8] I. Koltover, T. Salditt, J. O. Radler, C. R. Safinya, Science 1998, 281, 78.

[9] I. Koltover, T. Salditt, C. R. Safinya, Bioph. J. 1999, 77, 915.

[10] D. M. Kuntz, L. M. Walker, J. Phys. Chem. B. 2007 , 111, 6417.

[11] R. Martin-Rapun, M. Marcos, A. Omenat, J. Barbera, P. Romero, J. L. Serrano, J. Am. Chem. Soc. 2005, 127, 7397.

[12] M. Marcos, R. Martin-Rapun, A. Omenat, J. Barbera, P. Romero, J. L. Serrano, Chem. Mater. 2006, 18, 1206.

[13] N. Canilho, E. Kasëmi, R. Mezzenga, A. D. Schlüter, J. Am. Chem. Soc. 2006, 128, 13998.

[14] N. Canilho, E. Kasëmi, A. D. Schlüter, R. Mezzenga, Macromolecules 2007, 40, 2822.

[15] N. Canilho, E. Kasëmi, J. Ruokolainen, A. D. Schlüter, R. Mezzenga, Macromolecules 2007, 40, 7609.

[16] J. Ruokolainen, G. ten Brinke, O. Ikkala, Adv. Mater. 1999, 11, 777. 